ISSN: $1130-3743$

\title{
POTENCIALIDAD FORMATIVA DE LA LECTURA DE TEXTOS FENOMENOLÓGICOS DESDE LA PERSPECTIVA DE VAN MANEN
}

\author{
Formative potential of reading phenomenological texts \\ from the perspective of Van Manen
}

\section{Potentialité formative de la lecture de textes phénoménologiques selon Van Manen}

José Antonio Jordán Sierra

Universidad Autónoma de Barcelona. Facultad de Ciencias de la Educación.

Departamento de Pedagogía Sistemática y Social. Edificio G-6. 08193 Bellaterra

(Barcelona). Correo-e: josep.jordan@uab.es

Fecha de recepción: enero de 2008

Fecha de aceptación definitiva: abril de 2008

BIBLID [(1130-3743) 20, 2008, 125-150]

RESUMEN

Este artículo se centra en la gran potencialidad formativa encerrada en la experiencia de la lectura por parte de los educadores de un tipo singular de textos pedagógicos. Más en concreto, en este estudio se analiza la naturaleza virtualmente formativa de los textos fenomenológicos, que son -a su vez- los frutos finales de investigaciones fenomenológico-hermenéuticas tal como son entendidas y aplicadas por Van Manen y sus seguidores. Dichos textos son, esencialmente, distintos a muchos otros ofrecidos usualmente a los educadores en su proceso de formación. El contenido de esos textos, en efecto, es rico en elementos páticos, literarios, expresivos e incluso poéticos; algo que invita poderosamente a los educadores a cuestionarse sus esquemas y actitudes pedagógicas previas relacionadas con su mundo de la vida educativa cotidiana. En su parte tercera, el artículo aporta una investigación 
empírica práctica centrada en la experiencia lectora de una selección de textos fenomenológicos por parte de una muestra de profesores. Al final se concluye que todo apunta a confirmar que la potencialidad formativa encerrada en la lectura de ese tipo de textos aporta evidencias positivas y prometedoras en la línea deseada.

Palabras clave: lectura formativa, investigación fenomenológica-hermenéutica, textos fenomenológicos, formación de los educadores.

\section{SUMMARY}

This paper is focused on the great formative potential hidden in the experience of educators' reading a unique type of pedagogical text. Specifically, in this study we analyzed the potential formative nature of the phenomenological texts which are, at the same time, the final outcomes of phenomenologic-hermeneutic research as they are understood and applied by Van Manen and his followers. These texts are, essentially, different to many others that are usually offered to educators in their training process. Indeed, the contents of these texts are rich in phatic, literary, expressive and, even poetic elements, which powerfully invite educators to question their previous pedagogical attitudes towards their daily educational experience. The article also includes empirical research from a practical point of view, focusing on the reader's experience of meaningful phenomenological texts using a sample of teachers. Finally, the paper confirms that the formative potentiality hidden in the reading of this type of text brings positive and promising evidence, as thought.

Key words: formative reading, phenomenologic-hermeneutical research, phenomenological texts, educators' training.

\section{SOMMAIRE}

Cet article est centré sur la grande potentialité formative inhérente à l'expérience de la lecture faite d'un type singulier de textes pédagogiques par des éducateurs. On y analyse, en particulier, la nature virtuellement formative des textes phénoménologiques qui sont -à leur tour- les fruits finaux des recherches phénoménologique-hermenéutiques telles qu'elles ont été comprises par Van Manen et ses partisans. Ces textes diffèrent, essentiellement, des autres textes offerts habituellement aux éducateurs dans leur processus de formation. Leur contenu est, en effet, riche en éléments phatiques, littéraires, expressifs voire poétiques, ce qui mène les éducateurs à questionner leurs schémas et attitudes pédagogiques préalables vis-àvis de leur expérience éducative quotidienne. On y présente aussi les résultats d'une recherche empirique pratique sur l'expérience de lecture d'une sélection de textes phénoménologiques menée sur un échantillon de professeurs. Finalement, on conclut que tout semble confirmer que la potentialité formative enfermée dans la lecture de ce type de textes apporte des preuves positives et prometteuses dans la direction souhaitée. 
JOSÉ ANTONIO JORDÁN SIERRA

POTENCIALIDAD FORMATIVA DE LA LECTURA DE TEXTOS FENOMENOLÓGICOS

DESDE LA PERSPECTIVA DE VAN MANEN

Mots clef: lecture formative, recherche phénoménologique-herméneutique, textes phénoménologiques, formation des éducateurs.

\section{INTRODUCCIÓN}

La investigación fenomenológico-hermenéutica es, ciertamente, variada a la hora de aplicarla. Con todo, cuando se utiliza para escudriñar los aspectos esenciales del ámbito educativo destacan unas líneas prácticas más que otras. En nuestro caso, hemos elegido la orientación heurística propuesta por Van Manen, figura señera ampliamente reconocida hoy en día en este campo. Como veremos más adelante, toda investigación fenomenológico-hermenéutica que adopta el formato sugerido por dicho autor tiene como fruto o precipitado final la escritura de un texto fenomenológico; es decir, un relato que aglutina elementos cognitivos y emotivos, descriptivos e ilustrativos, en su intención de narrar singularmente la naturaleza o esencia de una dimensión de la experiencia propia del mundo de la vida pedagógica.

Tras esa aclaración inicial, puede entenderse mejor el cometido del estudio que abordamos en el presente artículo. El foco de interés del mismo, en efecto, se centra en la relación existente entre la experiencia de la lectura propia de educadores en formación o en ejercicio y el contenido sugestivo de los textos fenomenológicos. Más concretamente, el trabajo que presentamos pretende clarificar -teórica y prácticamente- en qué sentido y hasta qué punto leer tales textos fenomenológicos, bien nutridos de vivencias educativas, es potencialmente una actividad formativa para los educadores que realizan ese tipo de lecturas.

Para acometer este estudio nos ha parecido oportuno iniciar el recorrido con una alusión, breve pero clara, a ciertas tesis del profesor Larrosa (1996, 2003a, 2003b) en torno a la experiencia de la lectura como ejercicio eventualmente formativo. Las consideraciones agudas de este autor constituyen, a nuestro juicio, un buen primer punto de partida. Después, en un segundo momento, este trabajo se explaya coherentemente en la virtualidad formativa inherente a la naturaleza de los propios textos fenomenológicos, siguiendo ahora concretamente la línea de pensamiento de Van Manen -nuestro autor central- al respecto. En una tercera fase, este se centra en el análisis reposado de la constatación empírica del potencial formativo de una muestra de profesores que han leído una selección de textos fenomenológicos escritos y recogidos por el mismo Van Manen en uno de sus libros. Finalmente, se acaba concluyendo que todo lleva a pensar que, ciertamente y en condiciones plausibles, la lectura de dichos textos fenomenológico-pedagógicos contribuyen a formar y transformar los esquemas y actitudes previas de los educadores en relación a su propio mundo de la vida educativa cotidiana. 
JOSÉ ANTONIO JORDÁN SIERRA

POTENCIALIDAD FORMATIVA DE LA LECTURA DE TEXTOS FENOMENOLÓGICOS

DESDE LA PERSPECTIVA DE VAN MANEN

\section{UNA PRIMERA REFERENCIA}

Nos introducimos, pues, en el tema trayendo a colación algunos comentarios de nuestro primer autor de referencia, entresacados principalmente de su primer libro citado. Lo que le interesa a Larrosa es asegurar que la lectura sea realmente una experiencia que afecte a nuestro ser, en vez de quedarse en un ejercicio meramente superficial que sólo active el simple entretenimiento, la fugaz información, o el instrumental aprendizaje académico. Dicho lo mismo de manera más matizada:

Lo importante al leer no es lo que nosotros pensemos del texto, sino lo que desde el texto o a partir del texto podamos pensar de nosotros mismos. Si no es así, no hay verdadera lectura. Si lo importante fuera lo que nosotros pensamos del texto, habría erudición, filología. Tendríamos al final un texto esclarecido. Quizás habríamos aprendido algo que antes no sabíamos, pero a nosotros no nos habría pasado nada. Y de lo que se trata, al leer, es que a uno le pase algo [...] Lo que de verdad interesa es cuando el leer tiene efectos en uno, formándole y transformándole. Cuando la lectura es una experiencia que va de verdad, o como diría Gadamer, cuando es una verdadera experiencia, entonces afecta, en un sentido profundo, a lo que hay de más íntimo en el ser humano. Leer, cuando es más que cubrir un programa de estudios, más que un pasatiempo, más que un ejercicio cultural, es poner en cuestión eso que somos [...] Propongo algo así como entregarnos a un texto que dé que pensar, pero no sobre el texto, sino sobre nosotros mismos (Larrosa, 1996, 63-66).

Siguiendo este interesante filón, el centro de interés se sitúa en la lectura como experiencia de formación; ambición más codiciosa que la de buscar simplemente entender el significado externo de un texto, al tener lugar en este segundo caso un mero proceso de información, en donde el protagonismo es asumido fría y prioritariamente por el quehacer mental del lector. Dicho de forma contundente: un libro sólo es verdaderamente accesible a través de la propia experiencia vivida; sólo es posible leerlo en profundidad cuando no es sólo la inteligencia sino la vida personal toda la que se implica en el acto de lectura; sólo es formativo cuando no son sólo las ideas que lo tejen lo que se aspira a conocer sino cuando se busca ante todo llegar a vivirlo de verdad.

Por otro lado, en la lectura como formación lo importante no es tanto el texto sino la relación que tiene lugar con el texto. Una cita sobre Heidegger ilustra perfectamente esta idea:

El filósofo alemán «expresa de un modo muy hermoso la experiencia de esa relación, que no es de apropiación intelectual del contenido del texto sino la de escucha: "Hacer una experiencia con algo significa que algo nos acaece, nos alcanza; que se apodera de nosotros, que nos tumba y nos transforma [...] Hacer una experiencia quiere decir, por tanto, dejarnos abordar en lo propio por lo que nos interpela. Así es como podemos ser transformados por tal experiencia”» (op. cit., 19-20). 
En otro lugar de su obra, Larrosa abunda en esa dinámica propia de la formación que le acontece al lector cuando retorna distinto después de una genuina lectura:

La Bildung es un movimiento de ida y vuelta. El punto de partida es siempre lo propio, lo vital, lo familiar, que separa de sí mismo para ir a lo ajeno, lo desconocido [el texto], y regresar después, formado o transformado, al lugar de origen. Lo esencial de ese viaje de ida y vuelta es que constituyen un auténtica experiencia [...] Por eso, la Bildung no es una mera anexión mecánica y apropiadora de lo otro [del texto], sino que implica un devenir otro distinto al de antes y, en el límite, una verdadera metamorfosis (op. cit., 315).

El extenso libro elegido como punto de partida ofrece un sinfín de sugestivas referencias sobre estas vetas poco exploradas que van tras la experiencia lectora en su vertiente formativa. Para el cometido de nuestro estudio, sin embargo, parece suficiente acabar con una de ellas, dada su vinculación con la versión de Van Manen sobre los textos fenomenológicos y con el estudio práctico realizado con profesores en ejercicio de educación primaria. Esta es la cita a la que nos referimos:

Tanto la realidad, como nuestra propia vida, apunta Proust en su obra de madurez, sólo se nos dan en tanto que interpretadas o leídas. Y para aprender a leer el mundo y a leernos a nosotros mismos necesitamos la ayuda de los libros [...] A lo largo del relato, [se trata de aprender] a leerse a sí mismo y a descifrar el sentido del mundo en el que vive [...] Por eso dice Proust en su novela El tiempo recobrado que "cada lector, cuando lee, es el propio lector de si mismo". La obra del escritor no es más que una especie de instrumento óptico-un espejo- ofrecido al lector para permitirle discernir lo que, sin ese libro, no bubiera podido ver en sí mismo (op. cit., 93-94).

En relación con el interés que anima nuestro trabajo, puede ser útil recoger a continuación algunas de las frases-clave relacionadas con nuestra temática, presentes en el libro que andamos comentando.

\section{Cuadro 1. Frases clave Del profesor Larrosa}

Hacer una experiencia de genuina lectura quiere decir dejarnos abordar en lo propio por el texto que nos interpela (op. cit., 20).

Lo importante al leer es lo que a partir del texto podamos pensar de nosotros mismos" (op. cit., 63).

Eso de leer, cuando va en serio, tiene efectos en uno, formándolo o transformándolo (op. cit., 64).

El lector, cuando ciertamente lee, es el propio lector de sí mismo (op. cit., 94). 
JOSÉ ANTONIO JORDÁN SIERRA

POTENCIALIDAD FORMATIVA DE LA LECTURA DE TEXTOS FENOMENOLÓGICOS

DESDE LA PERSPECTIVA DE VAN MANEN

La obra del escritor no es más que una especie de instrumento óptico (un espejo) ofrecido al lector para permitirle discernir lo que, sin ese libro, no bubiera podido ver en sí mismo (op. cit., 94).

[En la experiencia lectora], la Bildung es un movimiento de ida y vuelta, e implica un devenir otro distinto al de antes; en el límite, es pues una auténtica metamorfosis (op. cit., 315).

Las historias ejemplares (el Edipo de Sófocles, el Quijote de Cervantes, el Fausto de Goethe, el agrimensor de Kafka...) son capaces de abrirnos nuevas posibilidades de vivir; son textos en relación a los cuales nos vemos compelidos a pensar en nuestras vidas; hasta el punto de podernos sacar de nosotros mismos y transformarnos o convertirnos en otros (op. cit., 473-474).

\section{LOS TEXTOS FENOMENOLÓGICOS Y SU INFLUJO EN LOS LECTORES}

En este apartado el objeto de atención es dar a conocer la naturaleza de los textos fenomenológicos, tal como son concebidos por el investigador y pedagogo Van Manen, y al mismo tiempo mostrar su potencialidad formativa en quienes los leen, especialmente en los educadores.

Aunque en los últimos años se está dando en nuestro país una paulatina difusión del pensamiento y de las obras de nuestro autor elegido, puede ser pertinente aquí realizar una presentación básica en torno a su figura y a su modo de entender la investigación pedagógica, con el fin de poder hacer así más inteligible lo que más adelante se comentará.

Van Manen, holandés de origen, recibió su formación docente en su país bajo la influencia de la Pedagogía Fenomenológica y Hermenéutica. Desde 1980 hasta el presente es Catedrático en Métodos de Investigación, Pedagogía y Estudios Curriculares del Departamento de Educación Secundaria de la Facultad de Educación en la Universidad de Alberta. Es el investigador principal del International Research Center of Phenomenology and Pedagogy, cuya finalidad es promover la investigación fenomenológica-hermenéutica a nivel internacional. Asimismo, es el fundador de la revista Phenomenology and Pedagogy, publicada durante diez años en la Universidad de Alberta, y vuelta a publicar recientemente con el título ahora de Phenomenology and Practice ${ }^{1}$. Van Manen es reconocido a nivel mundial como uno de los principales líderes en métodos de investigación en ciencias humanas de corte fenomenológico-hermenéutico; de modo que, como algunos han comentado con acierto, la orientación investigadora y la concepción pedagógica de Van Manen, centradas en la experiencia humana-educativa vivida, han supuesto -al

1. Puede consultarse esa revista entrando en http://phandpr.org/index.php/pandp/index. 
menos en los últimos veinte años- una alternativa a los paradigmas más convencionales (especialmente los tomados de las ciencias naturales) en el estudio de la infancia, el aprendizaje, el profesorado y la educación en sus variadas manifestaciones ${ }^{2}$.

Nuestro autor ha plasmado una visión, en gran parte original, de la pedagogía y de la investigación educativa, basándose en las tradiciones fenomenológica y hermenéutica de filosofía y pedagogía originadas principalmente a principios y mediados del siglo pasado en la Europa occidental ${ }^{3}$. Dotado de una sólida formación filosófica, especialmente en el campo de la fenomenología, Van Manen ha elaborado ciertamente una propia visión teórico-práctica de la metodología fenomenológico-hermenéutica, así como una orientación de cómo conviene aplicarla, fundamentalmente en el terreno pedagógico. Esa "fórmula metodológica", en buena parte singular, resulta inteligible con suficiente claridad tras leer con reposo y profundidad, especialmente, su libro básico en este filón: nos referimos al que, en traducción relativamente reciente al castellano, lleva por nombre Investigación educativa y experiencia vivida ${ }^{4}$. Con todo, conviene clarificar aquí que la fenomenología-hermenéutica de Van Manen -como él mismo aclara- tiene una decantación práctica, orientada al campo pedagógico, más que un interés prioritariamente filosófico ${ }^{5}$. Sin miedo a equivocarnos, podemos decir claramente que Van Manen

2. Destacamos los siguientes libros, traducidos al castellano: El tacto en la enseñanza. El significado de la sensibilidad pedagógica, Barcelona, Paidós, 1998; El tono en la enseñanza. Paidós, Barcelona, 2004; Investigación educativa y experiencia vivida, Barcelona, Idea-Books, 2003; Los secretos de la infancia. Barcelona, Paidós, 1999 (Levering y Van Manen). Puede resultar interesante entrar directamente en su propia página web: http://www.phenomenologyonline.com/MAX/.

3. Debemos pensar aquí, especialmente, en la "escuela de Utrecht" (Langeveld, Bollnow, Van den Berg, Buytendijk) y en la "escuela de Dilthey-Nohl" (Dilthey, Nohl, Schleiermacher, Gadamer), además de figuras centrales como Husserl, Heidegger, Marcel, Sartre, Merleau-Ponty, Ricoeur... y Lèvinas. Para mayor detalle son muy útiles los siguientes estudios: VAn MANEn (1978) y VAN Manen y Levering (2002).

4. La edición original, repetidamente reeditada, y citada en todo contexto en la mayor parte de las investigaciones de corte fenomenológico-hermenéutico, es la siguiente de 1990: Researching Lived Experience: Human Science for an Action Sensitive Pedagogy. State Unyversity of New York Press. Por otro lado, en nuestro país contamos con un primer estudio serio y extenso sobre la metodología peculiar de este autor, realizado con la finalidad de clarificarla, hacerla asequible y aplicarla a modo de muestra a una noción tan interesante como es la del "reconocimiento pedagógico"; estudio realizado por Raquel Ayala como Trabajo de Investigación previo a la Tesis Doctoral el pasado 2007 con el título siguiente: Introducción a la concepción y aplicación del método fenomenológico-hermenéutico de Max Van Manen, Universidad Autónoma de Barcelona (dirigido por mí mismo).

5. El propio autor concede que la fenomenología tiene unas ricas fuentes a las que conviene retornar una y otra vez para no perder los principios básicos rectores de este amplio movimiento filosófico. En él, en efecto, hay figuras señeras que son verdaderos faros orientadores; ahí se encuentran, por ejemplo, Husserl, Merleau-Ponty, Blanchot, Heidegger... y Ricoeur. Con todo, es indiscutible que se han ido produciendo diferentes corrientes que tienen sus propias orientaciones. El mismo Van Manen destaca las siguientes: a) Fenomenología trascendental, en la que sobresale, por supuesto, Husserl... junto con otros colaboradores e intérpretes suyos, tales como: Fink, Tymieniecka y Van Breda; b) Fenomenología existencial, asociada a Heidegger, Sartre, Merleau-Ponty, Marcel y otros; c) Fenomenología hermenéutica, 
JOSÉ ANTONIO JORDÁN SIERRA

POTENCIALIDAD FORMATIVA DE LA LECTURA DE TEXTOS FENOMENOLÓGICOS

DESDE LA PERSPECTIVA DE VAN MANEN

ha sido el pionero en el diseño de una metodología fenomenológico-hermenéutica que sea aplicable a campos en que primen las relaciones humanas, tales como la medicina, la enfermería... y, por supuesto, la pedagogía. Ya Vanderberg (1992) señalaba esta realidad, y, más recientemente, Barnacle $(2004,57)$ ha insistido en el mismo punto: lo más sustancial de la aportación de Van Manen ha consistido -dice este último- en haber realizado

la traducción de la fenomenología y la hermenéutica desde la arena filosófica al contexto de la investigación educativa; su modelo de investigación, basado en la noción de experiencia vivida, ha proporcionado una base para que los investigadores del ámbito de la educación puedan reflexionar sobre su propia experiencia personal como educadores, teóricos de la educación, directivos y responsables de la política educativa.

En este sentido, y sumarizando casi en exceso, puede decirse que su interés primordial consiste a fin de cuentas en llegar a "comprender" la experiencia vivida prerreflexivamente por los educadores y los menores en la cotidianidad e inmediatez propias del "mundo de la vida" educativa (Van Manen, 2003, 27). En esta línea, el fruto del proceso investigador fenomenológico-hermenéutico es un peculiar conocimiento reflexivo de específicas experiencias vividas que supone aportaciones de sumo interés, bien distintas a las contribuciones logradas por las ciencias sociales inspiradas en modelos científico-técnicos que prometen "explicar" y controlar comportamientos humanos. Con todo, en este marco diferenciador, tal y como apunta nuestro autor, las ciencias humanas fenomenológicas no son menos provechosas que las demás: sus aportaciones, en efecto, "contribuyen a "humanizar" la vida humana para ayudar a los seres humanos a ser cada vez más reflexivos sobre sus experiencias cotidianas y, así, poder estar más preparados para actuar

vinculada a filósofos clave, como el mismo Heidegger, Gadamer y Ricoeur; d) Fenomenología lingüística, en donde destacan Blanchot, Derrida y Foucault (aunque este último alguna vez no se perciba como fenomenólogo); e) Fenomenología ética, con figuras como Scheler, Lèvinas y Derrida. No cabe duda de que, para una visión profunda de la globalidad de estos movimientos, es casi indispensable acudir a la gran obra de SPiegelberg (1982), tan clara y sólida como amplia y matizada. Con todo, afirma Van Manen, "los implicados a nivel profesional -como es su caso- tienden a interesarse menos en el método propio de la filosofía fenomenológica que en su práctica y aplicación. De ahí que cuando exploramos la naturaleza de la investigación fenomenológica es conveniente distinguir inmediatamente entre la investigación realizada en este ámbito por los filósofos, en cuanto tales, y la investigación realizada por los implicados a nivel profesional". Después de enumerar diacrónica y sincrónicamente diferentes representantes de dicha fenomenología de la práctica, acaba situándose él mismo en esta línea teóricopráctica: "La fenomenología de la práctica se caracteriza por aplicar lo esencial del método fenomenológico a contextos profesionales, tales como la psicología clínica, medicina, pedagogía, enfermería..., y también en contextos en que priman asuntos del mundo de la vida diaria [...] En este campo cabe destacar inicialmente, por ejemplo, a Buytendijk dentro de la psicología clínica, y a Langeveld y Bollnow dentro de la pedagogía; y, actualmente, a Amadeo Giorgi, en el campo de la psicología, a Patricia Benner, en el terreno de la enfermería, y Van Manen, en el ámbito de la educación y de la pedagogía”. 
con tacto y acierto en determinadas situaciones [como, por ejemplo, las educativas]" (op. cit., 39).

Una vez ubicados básicamente en el contexto global del interés que singulariza el quehacer de nuestro autor, podemos ya adentrarnos en la naturaleza y cualidades de los textos fenomenológicos: una modalidad escrita que brota de la investigación fenomenológico-hermenéutica propia del autor escogido, y que resulta ser muy prometedora a la hora de realizar una experiencia de la lectura altamente formativa para los educadores.

\subsection{Un acercamiento a los textos fenomenológicos}

"Crear un texto fenomenológico - apunta claramente Van Manen- constituye el principal objetivo de ese tipo de investigación descrita"; un texto fenomenológico que, siendo el eslabón final del proceso investigador, consiste "en una narración discursiva sobre nuestras experiencias pedagógicas vividas en relación con los niños" (op. cit., 127). En otro lugar de ese mismo libro (centrado todo él en exponer el método fenomenológico-hermenéutico y en dar orientaciones sobre cómo ponerlo en práctica) se puede leer algo similar, pero más matizado: "El objetivo de la investigación fenomenológica es transformar la "experiencia vivida" de forma prerreflexiva en un texto que exprese su esencia, de tal modo que el efecto del texto sea a la vez un revivir reflejo y una aproximación reflexiva del significado de tal fenómeno: una noción por la cual el lector pueda cobrar vida con fuerza en su experiencia vivida" (op. cit., 45). El mensaje principal de estas citas es claro: el cometido primario de esta modalidad metodológica consiste en centrarse en las experiencias vividas objeto de estudio (por ejemplo, ¿qué significado otorgan los alumnos de secundaria a la vivencia de leer libros recomendados en las clases de literatura?). Ahora bien, una vez seguidos los pasos propios del método fenomenológico-hermenéutico, en la versión de este autor, lo fundamental es "lograr que ese aspecto del mundo de la vida, de la experiencia vivida por nosotros u otros, llegue a ser comprensible e inteligible de un modo reflexivo", para nosotros mismos y para los demás (op. cit., 142). Y eso se consigue a través de una buena narración o descripción de esas vivencias inmediatas y silenciosas a través del texto fenomenológico.

Líneas atrás se ha mencionado un ejemplo referido a la experiencia o significado dado a la vivencia de la lectura por los alumnos. Ahora podría pensarse en las múltiples experiencias que viven los docentes. Los profesores, en efecto, tienen una conciencia implicita de muchas experiencias vividas en su relación con los alumnos (por ejemplo, la esperanza que tienen en su mejora, la responsabilidad ante sus múltiples llamadas, la acogida practicada diariamente a todos y a los recién incorporados, etc.), pero necesitan textos fenomenológicos -O afines- que les presenten ese tipo de vivencias a nivel de conciencia reflexiva en un formato narrativo, de forma que leyendo dichos textos se sientan invitados e impulsados a 
re-conocer y a re-vivir esas experiencias o afines para reflexionar así sobre cómo podrían pensar y actuar en sus clases más convenientemente ${ }^{6}$. En otro ámbito paralelo, eso es lo que hacen por ejemplo las buenas novelas: desvelar en los lectores experiencias "ya vividas" o "potencialmente vividas" para facilitar que sean "saboreadas", "Contrastadas", "repensadas", "apropiadas"... hasta el punto de llegar así a estimular en el lector un proceso de formación con la ayuda de las filigranas encerradas en los relatos propios de la vida reflexiva ${ }^{7}$.

En esa línea que une la conciencia implícita del mundo de la vida cotidiana y la conciencia reflexiva que la tematiza, se encuentra el interés y la naturaleza de los textos fenomenológicos. En este sentido, quizás no sea redundante insistir en el cometido de esos textos, que tanto nos interesan para captar su valor formativo a través de su lectura.

Aunque paradójica, parece resultar cierta aquella frase del Principito de SaintExupéry: "Lo esencial es invisible para los ojos"; es decir, lo más cercano, vital y profundo de nuestras vidas queda normalmente retirado de nuestra primaria mirada escrutadora. Es en este sentido que Van Manen se atreve a decir que la experiencia vivida es, en principio, indecible e inefable, de manera que difícilmente podemos sacarla a la luz, con sus matices y sutilezas, sin la ayuda de otros que, con particular habilidad, expresan con sumo atino lo que nosotros quisiéramos decir, pero para lo cual no encontramos las palabras adecuadas" (op. cit., 130). Esa es la misión que cumplen de forma notoriamente acertada los grandes poetas... los destacados novelistas... y los buenos fenomenólogos. Es en esta línea donde emerge la feliz misión de los textos fenomenológicos, frutos finales -como ya se ha dicho- del proceso investigador fenomenológico-hermenéutico. Misión que queda más clara utilizando las propias palabras del autor: "Es "en" las palabras que tejen esos textos y "mediante" ellas que aquello -la experiencia vivida prereflexivamente- que traslucen, es decir, lo invisible, se torna visible" (op. cit., 147). De lo dicho aquí ya se puede ensayar una primera aplicación en relación a su

6. El autor distingue claramente entre dos tipos de "conciencia": la implícita y la reflexiva. Dejémosle hablar sobre esto: "En nuestra vida cotidiana se da una conciencia implícita, no temática, no reflexiva. Por ejemplo, "conciencia-yendo-en bici", "conciencia-hablando", "conciencia-ejerciendo -de-padre", "conciencia-ejerciendo-de-profesor", etcétera. No es todavía una conciencia "de” ir en bici, "de" hablar, "de" enseñar, "de" ejercer de padre, sino una conciencia tácita, una conciencia plena en esos sencillos actos cotidianos. Dicho de otro modo, vivir la vida significa "estar-en-el-mundo" como padre, como profesor... de forma activa, emocional; representa el hecho de ser consciente en el mundo de una forma no temática. Por el contrario, la conciencia reflexiva se encuentra continuamente alimentada por esta dimensión no reflexiva de la vida, a la cual convierte en su objeto temático. Como señala Merleau-Ponty (1975), la fenomenología constituye ese tipo de investigación en el campo de las ciencias humanas que debe aprovechar esta vida y darle expresión reflexiva" (op. cit., 58).

7. A este respecto, comenta: "El psicólogo fenomenólogo holandés Buytendijk (1961) destaca que se pueden extraer tal vez mayores aportaciones psicológicas de un gran novelista como Dostoyesky que de las típicas teorías académicas citadas en los manuales y publicaciones sobre ciencia social psicológica" (op. cit., 37). 
potencialidad en el campo de la experiencia vivida por los educadores en la realidad cotidiana de su tarea: puede decirse - por ejemplo- que, de algún modo, en los textos fenomenológicos está encerrada "la capacidad de "ver" esas vivencias pre-cognitivas y, en consecuencia, de prepararnos para una praxis consciente en el mundo de la vida pedagógica; de modo que, a partir de ellos, cabe decir que podemos ver cosas de nuestra práctica educativa que antes no podiamos ver" (op. cit., 146).

Naturalmente, para poder cumplir esa misión, los textos fenomenológicos han de ser peculiares descripciones relativas a la experiencia vivida. En este sentido, conviene clarificar que «el propósito de la investigación fenomenológico-hermenéutica no es el de llegar a formular un conocimiento generalizable, probando hipótesis y elaborando leyes causales". En su aspecto formal, un estudio fenomenológico no presenta informes o resúmenes conclusivos con los que suelen culminar aquellos otros convencionales. Más bien, su intención es "elaborar, en formato de texto, una descripción estimulante y evocativa de las acciones, conductas, intenciones y experiencias humanas tal como las conocemos en el mundo de la vida" (op. cit., 37). Por supuesto, los textos fenomenológicos difieren en ciertos aspectos de otros textos de ciencias sociales; entre otras cosas porque el conocimiento que se halla en ellos tiene, a la vez, dimensiones cognitivas y páticas, conceptuales y poéticas, informativas y formativas ${ }^{8}$.

En su discurso, estos textos "no sólo hablan a nuestra competencia intelectual sino a nuestras capacidades intuitivas prácticas, permitiéndonos así "ver" algo en una forma que enriquece nuestra comprensión de la experiencia de la vida cotidiana" $(1997,345)^{9}$. Si la dimensión cognitiva y conceptual es importante, también es verdad que aquella expresiva y literaria es fundamental. De ahí que los buenos textos fenomenológicos estén enriquecidos con vivos ejemplos, con el fin de "traer las experiencias vividas a la presente proximidad de forma que el lector pueda "conocerlas" cognitivamente y al mismo tiempo "sentirlas" no cognitivamente" (op. cit., 355). En esta línea, la utilización de historias y anécdotas juega un papel crucial en relación a la dimensión pática, poética, no cognitiva, formativa. El autor matiza, sin embargo, que las anécdotas, utilizadas de forma habitual a la hora de tejer los textos fenomenológicos, "no deben entenderse como simples ilustraciones que ayuden a aligerar o hacer más digerible un texto posiblemente nada fácil; las anécdotas deben entenderse, más bien, como instrumentos narrativos que posibilitan la comprensión de ciertas nociones que, de otro modo, fácilmente se nos escaparían" $(2003,132)$. Por otro lado, las anécdotas relativas a un fenómeno determinado suelen adquirir una significación de carácter ejemplar.

8. Ver la página web del autor: hhtp://www.phenomenologyonline.com/inquiry/68.html. 9. VAN MANEN (1997). 
JOSÉ ANTONIO JORDÁN SIERRA

POTENCIALIDAD FORMATIVA DE LA LECTURA DE TEXTOS FENOMENOLÓGICOS

DESDE LA PERSPECTIVA DE VAN MANEN

Dado que las anécdotas son concretas y se extraen de la vida misma (real o ficticia), se presentan, en efecto, como ejemplos o recomendaciones para actuar o ver las cosas de una determinada manera; en ese sentido, en la vida cotidiana es posible contar una anécdota como una respuesta con tacto, es decir, como un mensaje que intenta que el lector sienta o perciba una determinada verdad que sería difícil expresar en un lenguaje más transparente (op. cit., 136).

No en balde, "el fenomenólogo holandés Buytendijk describió una vez la fenomenología como la "ciencia de los ejemplos" (op. cit., 137). Más concretamente, y en relación al campo educativo, que es el que en realidad nos interesa: "las narraciones ricas en anécdotas son importantes para la pedagogía por lo bien que funcionan como materiales de casos vivenciales sobre los cuales es posible que los educadores lleven a cabo una fructífera reflexión pedagógica" (idem).

Con el fin de ilustrar más vivamente lo dicho en estos últimos parágrafos sobre la naturaleza de los textos fenomenológicos y, especialmente, sobre su dimensión literaria, pática, viva, expresiva y ejemplar, puede ser útil traer a colación un breve fragmento de un texto fenomenológico escrito por el propio Van Manen:

Una profesora de Lengua explica un incidente ocurrido en su clase con un alumno polémico:

Daniel tiene una forma provocadora de vestir, al estilo callejero. Posee un perfil que sorprende por su majestuosidad: labios sensuales, la cabeza afeitada, unos gestos fríos y elegantes, y una mirada orgullosa. Daniel destaca en nuestro pequeño instituto.

Es el primer día de clase y explico el programa de la Sala de Lectura. Daniel entra distraído en el aula, se sitúa directamente frente a mí y exclama: "Yo no leo".

"¿De verdad? -le replico-. Entonces la Sala de Lectura debería ser para ti una experiencia nada habitual". A medida que los alumnos empiezan a leer, la clase se tranquiliza. El silencio sustituye a las charlas y las discusiones en esa clase de noveno curso.

Observo que Daniel no tiene ningún libro. Está sentado en su sitio, repantigado en la silla y golpeando la mesa con el dedo índice. Examino detenidamente la estantería y selecciono unas cuantas novelas [...]. Me acerco despacio a su pupitre, me inclino y discretamente le susurro: “Oye, Daniel, ¿por qué no eliges el libro que te parezca menos aburrido... y a ver si lees unas cuantas páginas antes de que acabe la clase?".

Levanta sus ojos altivos y con un movimiento de la cabeza me perdona mi intromisión, y suspira: "Ya verée.

Pero yo le he dejado sobre la mesa los libros que había escogido con todo cuidado. Daniel sigue casi tumbado en la silla. Separa los libros con la mano, con una aparente indiferencia. Yo me vuelvo.

Unos minutos después, por el rabillo del ojo, veo que Daniel ha escogido The Freak, de Carol Matas.

"Sí, le he enganchado". Ahora debe ser el libro el que le atraiga. Tengo esperanza. Es posible que Daniel se convierta en un lector. 
¿Qué ocurre aquí? Una profesora ve-con mirada pedagógica- a un alumno que de forma deliberada actúa para que se le reconozca como diferente. El alumno pone a prueba la autoridad de la profesora. Sin embargo, ésta se niega a recurrir a su poder. La explicación que hace del incidente es suave y con sentido del humor, pero a la vez también respetuosa. Respeta el personaje que el alumno está creando; comprende que Daniel desea que se le reconozca. Y con este reconocimiento es como ella "aplica" el tacto. Parece que la profesora sabe exactamente hasta dónde acercarse al alumno y cómo mantener la debida distancia.

Pero ¿cómo sabe esta profesora lo que tiene que decir? ¿Cómo sabe qué distancia debe guardar? No se puede encontrar ningún conocimiento teórico, ni técnica concreta alguna sobre cómo actuar con tacto. La solicitud y el tacto pedagógicos son dos cosas que guardan una estrecha relación. La solicitud pedagógica es una capacidad reflexiva que nace de la reflexión pausada sobre las experiencias pasadas. Y ahora, en la inmediatez de tener que actuar en este momento, el énfasis se pone en el sentir qué es lo importante en esta situación concreta. El tacto en la enseñanza no es una simple destreza. Al contrario, se podría entender más bien como una "preparación para la improvisación". La solicitud no se puede describir de forma directa y sencilla, pero el tacto pedagógico se puede describir indirectamente, mediante ejemplos y anécdotas. Y esto es lo que he intentado hacer. Lo he descrito como una particular sensibilidad y sintonía con las situaciones (2004, 47 y ss.).

\subsection{Efectos formativos de la lectura de los textos fenomenológicos}

Llegados a este punto, se pueden ya señalar algunos de los efectos que tienen los buenos textos fenomenológicos en quienes los leen con sensibilidad, aunque algunos de sus influjos ya se han mencionado con anterioridad.

En primer lugar conviene tornar a la finalidad de los estudios fenomenológicohermenéuticos. Su cometido, desde luego, no es dar soluciones más o menos técnicas, potencialmente eficaces desde la lógica instrumental a posibles problemas educativos. Esto ya lo intentan otros enfoques más empíricos y usuales dentro de las ciencias sociales. Dicho lo mismo de forma clara: la aspiración de la fenomenología-hermenéutica es llegar a comprender el significado de fenómenos concretos del mundo de la vida (en nuestro caso, de la vida pedagógica). Expresando esto mismo con más distinción: el tipo de conocimiento encerrado en los textos fenomenológicos corresponde al esfuerzo por "comprender mejor y con mayor profundidad la experiencia vivida, con el fin de que, sobre la base de dicha comprensión, podamos ser capaces de actuar de un modo más reflexivo, con mayor tacto y acierto en determinadas situaciones (educativas)" (2003, 41). En este tipo de textos, pues, no encontrará el lector-educador explicaciones y reglas científicotécnicas, sino razones (de la inteligencia y del corazón) que le invitarán a cambiar actitudes y modos de actuar en su tarea de educar, que iluminarán y transformarán las motivaciones profundas que animan su manera de relacionarse pedagógicamente con niños y jóvenes. 
Desde esa visión, queda resuelta la cuestión del valor del conocimiento fenomenológico, criticado por algunos como inútil por su falta de pragmatismo desde el punto de vista de la razón instrumental. Por lo demás, dicho asunto ha sido mal planteado por sus censores. Así lo pone de manifiesto el propio Van Manen: "Parafraseando a Heidegger, la cuestión más importante no es "¿podemos hacer algo con la fenomenología?", sino aquella otra, mucho mejor planteada: "si nos interesamos en la fenomenología profundamente, ¿puede ella hacer algo con nosotros?"” (op. cit., 65). La respuesta a esta última pregunta es, por supuesto, afirmativa. Veamos a continuación algunos de los efectos que sobre nosotros puede ejercer el conocimiento fenomenológico presente en dichos textos:

En primer lugar, ese conocimiento peculiar "nos habla, dirigiéndose a nuestras sensibilidades cognitivas y no cognitivas" (1997, 345); más aún, el buen texto fenomenológico pretende encantarnos, trayendo la experiencia de forma especialmente vívida a nuestra presencia, haciéndonos así fácil el poder reflexionar fenomenológicamente sobre ella (op. cit., 353). En la medida que seamos sensibles, dejando que el texto nos hable, que su significado más profundo tenga un efecto no cognitivo sobre nosotros como lectores (op. cit., 359), "seremos, literalmente, impresionados y afectados por él, no pudiendo alejarnos demasiado de su cualidad bechizadora" (op. cit., 364). Todo esto ocurre en los lectores en virtud de la textura pática, literaria, expresiva y viva del texto, al referirse tal conocimiento a la experiencia vivida propia del mundo de la cotidianidad, lo que hace que haya en el mismo tanta o mayor dosis de "poesía" como de "prosa". Eso explica que, con frecuencia, el poder narrativo del texto pueda llegar a ser para el lector "más atractivo, conmovedor y vibrante en el plano emocional, que la vida misma" $(2003,145)$.

Ese clima "pático" ${ }^{10}$ predispone a los lectores a sentirse interpelados por los mensajes experienciales del texto, a ser llamados a vivir de forma más plena como educadores, gracias al hecho de redescubrir en la lectura nuevas posibilidades existenciales relativas a qué debe ser o hacer un verdadero profesor. En relación a este punto, Van Manen no sólo apunta que el texto fenomenológico "devuelve al lector a su propio mundo de la vida", facilitándole el re-conocerse de forma reflexiva y, consecuentemente, invitándole a mejorarse como educador de modo pleno (op. cit., 65 y 95). El autor va más allá todavía cuando sostiene que "la descripción propia del texto fenomenológico conlleva una fuerza moral. (op. cit., 31); esto es, que apunta siempre a lo que es la esencia, naturaleza o deber ser de "algo": por ejemplo, de lo que es "ser un (buen) profesor" o, por hacer otra mención, de lo que es "ser una (buena) relación educativa responsable». Exposición ésta de lo "excelente" que se convierte para el lector en una potente llamada a la que se ve impelido a responder de manera afirmativa; respuesta que supone, a su vez, una transformación de su forma habitual de pensar y de actuar en su práctica educativa.

10. Ver sobre esta cualidad pática el artículo de VAN MANEN y LI (2002). 
En otro lugar, Van Manen concreta todavía más el efecto transformador de un texto fenomenológico, escrito -recordémoslo- con acentos no sólo descriptivostemáticos sino también y abundantemente páticos-expresivos. Veamos algunas de estas citas relevantes:

El poder de los textos fenomenológicos reside en la resonancia (reverberación fenomenológica) que sus palabras pueden efectuar en nuestra comprensión, incluidas aquellas zonas de comprensión que son de algún modo pre-discursivas y pre-cognitivas y, en ese sentido, menos accesibles al pensamiento conceptual e intelectual. El uso creativo de las palabras que tejen los textos puede dar lugar a imágenes evocadas que pueden movernos: in-formarnos para formarnos, dejando así un efecto sobre nosotros. Cuando esto ocurre, dice Gadamer (1996), entonces el lenguaje (del texto) nos toca en el alma. O como Bachelard (1964) apunta: la reverberación provoca un cambio de ser, de nuestra personalidad (p. xviii) $(1996,58)^{11}$.

Pocos comentarios necesita esta sustanciosa cita, dado que habla de manera suficientemente rica por sí misma. Tan sólo convenga, quizás, parafrasear el potencial formativo en profundidad que tienen los textos fenomenológicos en la medida que son capaces de hacer resonar con fuerza la experiencia vivida narrada hasta llegar a "tocarnos en el alma" y a "cambiar nuestra misma personalidad". Volveremos, no obstante, sobre esto en la parte más práctica de este trabajo.

No deseando extendernos más de lo necesario, nos limitaremos a traer aquí otra cita capital para el filón que nos interesa:

Un texto fenomenológico nunca debe ser leído meramente por su mensaje superficial. El sentido epifánico del texto está directamente relacionado con el significado de la vida que la fenomenología intenta evocar [...] Experimentamos su cualidad epifánica cuando un texto, repentinamente, nos habla, cuando se dirige a nosotros de forma que valida nuestra propia experiencia, cuando transmite una comprensión de la vida que conmueve nuestras sensibilidades, cuando pulsa las cuerdas de la unidad de nuestro ser. El texto fenomenológico hace que el mundo de la vida se dirija a nosotros y nos lance una llamada a pensar y a sentir sobre él. El texto fenomenológico nos empuja, así, a experimentar el significado de la vida reflexivamente a nivel de conciencia pre-reflexiva y a nivel de significado reflexivo en lo que se refiere a nuestro papel en la vida [jtambién pedagógica!] (1997, 366-367).

El buen texto fenomenológico, ciertamente, tiene una gran potencialidad transformadora al permitirnos percibir "una comprensión intuitiva del significado de una determinada experiencia vivida; algo que puede llegar a ser tan fuerte o llamativo que es capaz de conmovernos en el núcleo de nuestro ser" (ídem). Esto, en buena medida, se debe a que lanza una llamada sobre el lector invitándole con fuerza no sólo a pensar sobre la experiencia vivida directa o vicariamente, sino

11. VAN MANEN (1996). 
JOSÉ ANTONIO JORDÁN SIERRA

POTENCIALIDAD FORMATIVA DE LA LECTURA DE TEXTOS FENOMENOLÓGICOS

DESDE LA PERSPECTIVA DE VAN MANEN

también a sentir la vida encerrada en la descripción del texto, sin quedarse en el significado frío de las palabras del relato, que podrían ser leídas simplemente en clave intelectual o conceptual.

\subsection{La lectura fenomenológica desde el mundo de la novela}

Podría acabarse aquí esta parte, sin agotar, por supuesto, su contenido ${ }^{12}$. Sin embargo, parece sensato ultimar estas consideraciones, trayendo a colación algunas observaciones paralelas que nuestro autor hace en una publicación centrada en las virtualidades de la buena novela. De este modo, además, podemos vincular lo dicho sobre los textos fenomenológicos con ciertos comentarios del principio de este trabajo sobre la contribución del profesor Larrosa a la lectura como formación.

Sin dejar de lado el trasfondo fenomenológico, Van Manen dedica un artículo a cómo las novelas nos educan. Refiriéndose a la lectura de la novela Cisco el rata, de Piet Bakker, comenta: "La lectura de esta novela influyó en mi adolescencia y, después también, en mi deseo de llegar a ser profesor $(1985,177)$. La novela en cuestión narra una inspirada historia sobre un profesor y sus fracasos y éxitos con un muchacho de un barrio marginal, Cisco, de sobrenombre El rata. A raíz de esa experiencia, Van Manen se pregunta: “¿Cuál es el poder de este texto?», y “¿Por qué pueden educar las novelas?”. Y pasa a dar algunas contestaciones a sus cuestiones, aquí sólo recogidas sucintamente.

Gracias a la cualidad pática del texto, "la buena novela nos encanta, porque leer tiene mucho de mágico, de absorbente, de encantador. La novela ejerce su reclamo y en ese momento puede educarnos, transformándonos de un modo especial" (op. cit., 177). Lo que la literatura de ficción nos revela "es un saber que es más bien una vida. Indirectamente, vicariamente, venimos a conocer lo que no podemos comprender, ver, oír o sentir, de un modo directo o conceptual" (op. cit., 178). En realidad, "la fuerza de una gran novela es que nos proporciona una valiosa y poderosa experiencia humana" (op. cit., 180). Ciertamente, "la experiencia del texto es una sutil pedagogía: al abrir un libro, yo mismo me abro a él: me hago vulnerable; me arriesgo a experimentar pensamientos y sentimientos que posiblemente no me dejen ser el mismo de antes" (op. cit., 184). "Así, por ejemplo, la experiencia de leer a Dostoyeski invade mi mente y deja su poso, de modo que -aun después de dejar el libro un tiempo- yo todavía estoy empapado por los sentimientos despertados por su lectura. De ahí en adelante, yo no nunca seré el mismo otra vez. $\mathrm{Al}$ abrir la novela, al igual que un amante, ella abre mi interior y toca mi corazón en una forma que pocas otras experiencias pueden hacerlo" (op. cit., 185). "Así, la gran novela ofrece una doble, pero sutil, pedagogía: cultiva tanto

12. En este sentido, el lector interesado en esta temática puede consultar con provecho el último artículo de VAN MANEN (2007), publicado en la nueva revista que él dirige: Phenomenology $\varepsilon$ Practice. 
nuestra vida pre-reflexiva como nuestra vida reflexiva. Leer es, así, llegar a ser experimentado. Y debido a las consecuencias reflexivas de la experiencia de la lectura, ello implica llegar a conocerse a uno mismo" (idem).

Como puede advertirse, Van Manen abre de este modo el potencial formativo de la lectura a un campo más extenso que el propio de los textos fenomenológicos en su puridad. Según se desprende de sus reflexiones sobre la influencia humana de las buenas novelas, la virtualidad formativa sobre los educadores la pueden poseer también diversos tipos de lecturas -tales como, por ejemplo, biografías, relatos, novelas, etc.- con la condición de que, además de su esencial e intrínseco valor pedagógico de fondo, contengan características básicas como las señaladas más arriba en el caso de los textos fenomenológicos; o dicho esto último de forma más concreta: en su estructura narrativa deben estar incluidos de forma inherente elementos no sólo cognitivos, conceptuales e informativos, sino también páticos, expresivos, literarios y formativos.

Al igual que hicimos en la primera parte, también ahora puede ser útil recoger algunas de las frases-clave relacionadas con nuestra temática, esparcidas en las páginas anteriores por Van Manen:

\section{Cuadro 2. Ideas Clave del Profesor Van Manen}

En los textos fenomenológicos está encerrada "la capacidad de "ver" esas vivencias pre-cognitivas y, en consecuencia, de "prepararnos" para una praxis consciente en el mundo de la vida pedagógica; de modo que, a partir de ellos, cabe decir que podemos ver cosas de nuestra práctica educativa que antes no podiamos ver, $(2003,146)$.

Las narraciones ricas en anécdotas son importantes para la pedagogía por lo bien que funcionan como materiales de "casos vivenciales" sobre los cuales es posible que los educadores lleven a cabo una fructífera "reflexión pedagógica" $(2003,137)$.

El tipo de conocimiento encerrado en los textos fenomenológicos corresponde al esfuerzo por "Comprender mejor y con mayor profundidad la experiencia vivida, con el fin de que, sobre la base de dicha comprensión, podamos ser capaces de actuar de un modo más reflexivo, con mayor tacto y acierto en determinadas situaciones (educativas)" (2003, 41).

"La descripción propia del texto fenomenológico conlleva una fuerza moral" (2003, 31); esto es, apunta siempre a lo que es la esencia, naturaleza o deber ser de "algo": por ejemplo, de lo que es "ser un (buen) profesor". Exposición ésta de lo "excelente" que se convierte para el lector en una potente llamada a la que se ve impelido a responder de manera afirmativa; respuesta que supone, a su vez, una transformación de su forma habitual de pensar y de actuar en su práctica educativa. 
JOSÉ ANTONIO JORDÁN SIERRA

POTENCIALIDAD FORMATIVA DE LA LECTURA DE TEXTOS FENOMENOLÓGICOS

DESDE LA PERSPECTIVA DE VAN MANEN

El poder de los textos fenomenológicos reside en la resonancia (reverberación fenomenológica) que sus palabras pueden efectuar en nuestra comprensión, incluidas aquellas zonas de comprensión que son de algún modo pre-discursivas y pre-cognitivas $\mathrm{y}$, en ese sentido, menos accesibles al pensamiento conceptual e intelectual. Cuando esto ocurre, dice Gadamer (1996), entonces el lenguaje (del texto) nos toca en el alma. O como Bachelard (1964) apunta: la reverberación provoca un cambio de ser, de nuestra personalidad (p. xviii) $(1996,58)$.

Experimentamos su cualidad epifánica cuando un texto, repentinamente, nos habla, cuando se dirige a nosotros de forma que valida nuestra propia experiencia, cuando transmite una comprensión de la vida que conmueve nuestras sensibilidades, cuando pulsa las cuerdas de la unidad de nuestro ser. El texto fenomenológico hace que el mundo de la vida se dirija a nosotros y nos lance una llamada a pensar y a sentir sobre él. El texto fenomenológico nos empuja, así, a experimentar el significado de la vida reflexivamente a nivel de conciencia prereflexiva y a nivel de significado reflexivo en lo que se refiere a nuestro papel en la vida (jtambién pedagógica!) (1997, 366-367).

La experiencia del texto es una sutil pedagogía: al abrir un libro, yo mismo me abro a él: me hago vulnerable; me arriesgo a experimentar pensamientos y sentimientos que posiblemente no me dejen ser el mismo de antes (1985, 184).

\section{APROXIMACIÓN PRÁCTICA: LECTURA DE UN TEXTO FENOMENOLÓGICO POR LOS PROFESORES}

En este tercer apartado damos a conocer la experiencia vivida por un grupo de profesores de primaria que leyeron en el tercer trimestre del curso 2006-2007 una serie de textos fenomenológicos escritos por el propio Van Manen, recogidos en su libro El tono en la enseñanza. Este libro es peculiar porque no tiene un formato monográfico, sino que está elaborado a partir de un conjunto variado de diversos textos fenomenológicos originarios, fruto de investigaciones fenomenológico-hermenéuticas del autor ${ }^{13}$.

Los profesores que constituyen la muestra de nuestro estudio eran pues, en este caso, alumnos universitarios de un grupo de la tarde (titulación de Pedagogía), inscritos en una asignatura sobre Ética profesional docente que impartía y que se prestaba a este tipo de lecturas. Dichos profesores, todos ellos en ejercicio, eligieron mayoritariamente y con agrado leer ese libro de Van Manen, aunque podían optar -si lo deseaban- por leer otro distinto entre varios sugeridos. Concretamente,

13. A modo ilustrativo, mencionamos algunas nociones más interesantes de esos textos recopilados en dicho libro: "La solicitud y el tacto pedagógicos", "Comprensión del mundo de posibilidades del niño", "La experiencia de la curiosidad y el asombro del niño", "Ver al niño desde una mirada pedagógica", "La importancia del reconocimiento", "¿Cómo experimentan los niños nuestra presencia?", "El poder de la atmósfera pedagógica", "La esperanza pedagógica”. 
fueron 12 los profesores que eligieron este libro de Van Manen, mientras que otros 3 decidieron leer otro alternativo ${ }^{14}$. A todos los que decidieron leer ese compendio de textos fenomenológicos que conforman el libro El tono en la enseñanza se les propuso una serie de breves y sencillas sugerencias sobre cómo enfrentarse a la lectura del mismo. Concretamente, las orientaciones fueron las siguientes:

a) No se os pide nada concreto, en la línea de la evaluación típica de un libro obligatorio o recomendado. Es decir, deseo que leáis el texto con libertad, sin pensar en aspectos pragmático-académicos como, por ejemplo: entender lo que se relata, realizar sintesis de las ideas principales, estudiarlo a un nivel meramente académico, etcétera. Os lanzo la invitación, más bien, de hacer una lectura desde vuestra mirada personal-profesional, como educadores que sois.

b) En este sentido, lo que os sugiero es que leáis el libro en abierto diálogo con las experiencias y comentarios que el autor del libro os brinda de forma viva.

c) Durante la lectura, o al final de ella, conviene que vayáis escribiendo lo que sentís, pensáis, experimentáis, juzgáis, aplicáis, etcétera, a raíz de lo leído en actitud reposada, sin las presiones académicas convencionales.

d) Al final de vuestros escritos podéis entregar unas notas de pocas páginas en las que recojáis lo que más os haya influido en ese proceso de lectura.

Durante más de dos meses dichos profesores tuvieron tiempo para hacer esa lectura sugerida, en tiempos tranquilos al margen de los propiamente lectivos. Aunque la muestra no era numerosa, el resultado tangible fue más de un centenar de páginas escritas con calor y profundidad. Nuestra labor posterior consistió en extraer las frases y fragmentos más interesantes y significativos relacionados con la lectura como experiencia formativa. El resultado final consistió en diez nutridas páginas de este tipo de material seleccionado extraído.

Creemos conveniente matizar en este momento que -sin excepción algunatodos los profesores que leyeron el citado libro (compendio de ejemplos de textos fenomenológicos) se implicaron activamente en el proceso lector una vez animados y orientados por nuestras sugerencias, de forma que -a la vista de sus reflexiones escritas- cada uno de ellos, con su sello personal, entabló un verdadero diálogo con los textos incluidos en el libro, salpicando los escritos que hacían mientras realizaban su lectura con abundantes y ricos comentarios y ejemplos propios, vinculados a su vida profesional educadora. En coherencia con lo dicho, podemos asumir, aunque sólo sea a través de esta vía indirecta, que los textos les

14. Ese segundo libre de posible elección era concretamente el de Hansen (2001) Llamados a enseñar. Barcelona, Idea-Books. 
habían encantado en su sentido más hondo, interpelándolos y haciéndolos pensar y repensar con visible profundidad sobre su ser y quehacer como educadores.

Hecha esta breve introducción, a continuación nos limitaremos a recoger, por interés y espacio, algunos -entre los más relevantes- de los comentarios de los profesores lectores, a la vez que haremos una serie de consideraciones propias con el fin de interpretar su valor en la línea perfilada en este estudio. La finalidad de cuanto sigue en esta parte no casa con el modelo estándar, sobre todo "empírico", que tiene la pretensión más o menos explícita de "demostrar" algo concreto. La finalidad de la interpretación del material recogido en nuestro estudio práctico, en coherencia con la filosofía de fondo que anima la misma metodología de nuestro autor, consiste más bien, por nuestra parte, en "mostrar" que, ciertamente, los textos fenomenológicos parecen tener una visible potencialidad formativa cuando son leídos por los educadores sin objetivos académicos de corte claramente utilitario. Veamos algunos de estos fragmentos elegidos:

Al leer los comentarios de Van Manen se me iluminan y se me hacen más conscientes cosas que yo ya sabia antes de algún modo, incluso que de alguna manera ya hacía, pero sobre las cuales no me había planteado antes el porqué. Simplemente creía que se tenían que hacer así, y ya está. El hecho de haber leído este libro me ha activado este sentimiento reflexivo de encontrar el porqué de cada cosa que debo hacer. Y si sé por qué se ha de hacer algo, es más fácil ir mejorándome como profesor (Y8).

Ese fragmento, al igual que otros bastante similares, viene a ratificar lo que Van Manen ya nos había hecho notar en uno de sus libros: en los textos fenomenológicos está encerrada "la capacidad de "ver" esas vivencias pre-cognitivas y, en consecuencia, de "prepararnos" para una praxis consciente en el mundo de la vida pedagógica; de modo que, a partir de ellos, cabe decir que podemos ver cosas de nuestra práctica educativa que antes no podiamos ver, $(2003,146)$.

Me ha gustado la frase de Van Manen "La educación de los niños se convierte en auto-educación". Ese pensamiento me ha hecho más consciente de mi capacidad de influir en los niños con palabras, conductas... Por eso también hemos de reflexionar más en quiénes somos, en cómo somos, en cómo actuamos... Este hecho de reflexionar sobre nosotros mismos nos hace cambiar al mismo tiempo que nos auto-conocemos más. Personalmente, he de decir que el libro de Van Manen que he leído me ha ayudado a ser más consciente de mi forma de ser y de comportarme en mi relación diaria con los niños de mi clase (P2).

En una línea no muy diferente a la propia del fragmento anterior, esta profesora insiste en el efecto que ha tenido en ella la lectura del texto fenomenológico: ahora siente la necesidad de auto-cuestionar a fondo su ser y quehacer docente; reflexión que le encamina a cambiar su experiencia práctica cotidiana como maestra. Veamos algo parecido, pero dicho con más intensidad: 
Cuando pienso en mi responsabilidad como educador y miro a los niños con una mirada pedagógica, ahora intento pensar en cada momento cuál es la manera correcta de hablar, qué he de decir, qué he de hacer, cómo conducirme, qué manera es la mejor en cada momento para que estén atentos, para exigirles según convenga... Pienso que eso se debe a la lectura de este libro, que me está pidiendo todo eso. Antes me preguntaba esas cosas a veces, sin exigirme tanto. Ahora me cuesta hacer cualquier cosa cuando estoy con los niños sin pensar en El tono en la enseñanza (S6).

En el comentario anterior puede verse meridianamente cómo el texto ha interpelado al lector, transformándolo y convirtiéndolo en otro distinto a quien era antes, al menos en su personalidad profesional. Un cambio debido por lo demás -y esto es fundamental- a la fuerza moral inherente a todos los buenos textos fenomenológicos, como apunta Van Manen $(2003$, 31). La lectura de esos textos compendiados en el libro leído parece haberse convertido para este profesor en una potente llamada, convencidamente interiorizada, que le invita a responder afirmativamente a lo que "debe hacer" como buen docente; lo que supone, a su vez, una cierta metamorfosis sobre su forma habitual de pensar y actuar en su práctica educativa. Fijémonos en el siguiente fragmento escogido cómo los componentes literarios, no cognitivos, páticos y expresivos, presentes -por ejemplo- en las anécdotas y el lenguaje cautivador de los textos leídos por nuestros profesores llegan, en ese caso, a tocar el alma, como gustaba decir a Gadamer:

La anécdota de las estrellas de mar, salvadas por aquel joven comprometido con la vida, que narra Van Manen... me ha llevado a preguntarme: ¿podré yo sacar adelante algunos niños que aparentemente no tienen mucho futuro educativo? A veces, es verdad que al ver que son muchos, cada uno con sus circunstancias y algunos con problemas serios, viene la tentación de rendirse y atender al grupo, pensando "¡sálvese quien pueda!». Sin embargo, después de leer esa historia y los comentarios de Van Manen, se me ha metido dentro una inquietud: ¿no vas a poder hacer algo más por fulanito y menganito? Y me cuesta no responder con esperanza: ¡Quizás si les das un plus de dedicación, alguno de ellos saldrá de su penosa situación! (E8).

Podría extenderme mucho más trayendo aquí numerosos comentarios espontáneos de los lectores de esos textos fenomenológicos, pero el espacio pide poner, de momento, un punto final. Con todo, traigo todavía a colación un par de fragmentos de una profesora lectora, que tienen que ver con el tema de la "utilidad" o -¿mejor?- del provecho pedagógico que puede suponer leer este tipo de textos por los educadores; o dicho de otro modo, parafraseando la cuestión que Van Manen pone en boca de Heidegger: "La cuestión más importante... y bien planteada es: "si nos interesamos en la fenomenología profundamente, ¿puede ella hacer algo 'con' nosotros?”, (2003, 65). Veamos:

Si me preguntaran si es útil un libro como éste, evidentemente diría que sí. Alguno puede pensar que son "cuatro anécdotas" con algunas reflexiones, pero yo creo 
JOSÉ ANTONIO JORDÁN SIERRA

POTENCIALIDAD FORMATIVA DE LA LECTURA DE TEXTOS FENOMENOLÓGICOS

DESDE LA PERSPECTIVA DE VAN MANEN

que va más allá. Hay mucha literatura científica sobre la educación que, aunque pueda ser necesaria, frecuentemente se aleja de la práctica diaria. Con una narración como la de este libro una puede sentirse inmediatamente identificada en los numerosos ejemplos que nos retratan y dan que pensar sobre la propia práctica (E11).

Van Manen acaba esta parte comentando la importancia de leer textos significativos sobre niños, sobre todo para los educadores, ya que aportan a los docentes una visión que hace reflexionar sobre la propia práctica como profesores. La verdad es que mientras leía este libro iba pensando en la opción de dejar un ejemplar de este libro sobre la mesa de la sala de profesores. Encuentro que es un libro que hace reflexionar muchísimo sobre la propia práctica docente, y estoy convencida de que más de un profesor/a se animará a abrirlo y leerlo (N3).

¿No hablan por sí mismos estos comentarios? ¿Puede, pues, la fenomenología -los textos fenomenológicos concretamente- "hacer algo con nosotros"?

\section{ANÁlISIS FINAL}

La primera consideración hace referencia a la abundancia de comentarios de los profesores de nuestra muestra que van en la línea de la lectura como experiencia de formación, en sintonía con lo apuntado por Larrosa al comienzo de este artículo y -especialmente- con lo propuesto por Van Manen sobre la potencialidad formativa de la lectura de textos fenomenológicos. En este sentido, cabe decir que, aunque son géneros escritos inconfundibles, de hecho los textos fenomenológicos tienen muchos elementos páticos, literarios, expresivos, e incluso poéticos, que los hacen en gran parte semejantes a otros textos propios del ámbito de la literatura y de otros campos próximos; textos estos últimos en los que se centra la experiencia de la lectura de Larrosa, y que el mismo Van Manen, como hemos visto en un artículo suyo sobre el poder educativo de la novela, también aborda y aplaude.

Aunque es imposible trasladar a estas páginas esa abundancia de comentarios significativos de nuestros profesores, lo que sí puedo intentar es mencionar algunas "frases-clave" que se repiten continuamente. Cito las más relevantes:

\section{CuAdro 3. Reflexiones ClaVe De los PROFESORES lectores}

Antes de leer este libro no me interrogaba apenas sobre mi práctica educativa. Me centraba en preparar actividades para los alumnos. Nunca me preguntaba sobre las vivencias concretas de cada niño singular.

El libro me ha recordado aquel maestro que admiro, que tanto se acerca al buen profesor que el autor comenta en el texto. A base de reflexión y experiencia, ime gustaría parecerme a ese excelente profesor! 
En magisterio aprendí muchas teorías educativas y me empapé de métodos y técnicas. Llegué a creer que eso era la panacea para mi futura práctica. Ahora veo que no es así. Me faltaban grandes dosis de tacto y solicitud pedagógica. Ahora, ante situaciones no fáciles, en balde rebusco en mi mente aquellas teorías de la universidad; a veces, casi pienso que el mejor uso de aquellos libros es darle con alguno de ellos en la cabeza a los que me causan problemas. Ahora veo que lo que me piden los niños es saber reaccionar con tacto.

Eso es algo que, de algún modo, siempre he tenido en mente, pero nunca lo había experimentado de una forma tan intensa como abora (después de esta lectura).

La lectura de este libro ayuda a reflexionar mucho sobre la propia práctica. Por poco críticos que seamos, seguro que encontramos cosas que no concuerdan con lo que los relatos del autor nos hacen descubrir.

Lo que he leído sobre qué significa "Ser" profesor me estimula ahora a mía ser una verdadera maestra en la relación diaria que tengo con mis alumnos.

Me ha gustado eso que dice Van Manen y, sobre todo, me ha hecho cambiar muchos esquemas mentales.

Si nos paramos a pensar, después de leer el texto, hay bastantes cosas que deberíamos mejorar como profesores, y esto es algo que deberíamos plantearnos muy seriamente.

Habiendo llegado aquí, conviene subrayar -en primer lugar- que los textos fenomenológicos (como el ofrecido a esos educadores) conducen a una experiencia lectora peculiar, diferente a la que fomentan los textos convencionales que acostumbran a leer usualmente los profesores en formación o en ejercicio; experiencia lectora atípica originada por la singularidad del conocimiento encerrado en dichos textos ${ }^{15}$. Ese conocimiento singular, fruto de la investigación fenomenológica, posee la virtualidad de poner al lector en estrecho contacto con la "experiencia vivida" por uno mismo o por otros (en situaciones análogas); contacto que permite la posibilidad de ir llenándose de Erfabrungen (experiencias de vida

15. "El objetivo de la reflexión fenomenológica -dice VAN MANEN- es el logro de un conocimiento personal y formativo. La importancia práctica del texto fenomenológico y de la comprensión (a que da lugar) no consiste en ofrecer útiles teorías y técnicas que uno pueda aplicar para solucionar problemas en situaciones prácticas. La conexión entre el conocimiento fenomenológico y la práctica no es una relación técnica. La fenomenología no proporciona "información" en el sentido habitual del término. Más exactamente, la importancia práctica del conocimiento fenomenológico es, por su naturaleza, formativa: dicho conocimiento, en efecto, aumenta nuestra sensibilidad, contribuye a la mejora de nuestro tacto en las relaciones humanas (educativas), y nos proporciona formas páticas de comprensión personalizadas, situacionales, relacionales y enactivas" (http://phenomenologyonline. com/inquiry/68.html). 
JOSÉ ANTONIO JORDÁN SIERRA

POTENCIALIDAD FORMATIVA DE LA LECTURA DE TEXTOS FENOMENOLÓGICOS

DESDE LA PERSPECTIVA DE VAN MANEN

acumuladas), pudiendo así darse "un efecto transformador en nuestro ser" ${ }^{16}$ (2003, 192) y llegar a "ser experimentados" o, lo que es lo mismo, poseedores de una "madura sabiduría de la práctica de vivir (pedagógicamente, en nuestro caso), como resultado de haber vivido la vida (educativa) en profundidad" (op. cit., 50). Dicho llanamente: el conocimiento propio de todo buen texto fenomenológico posee per se una alta virtualidad formativa ${ }^{17}$, si se lo compara con otros conocimientos habituales de muchos textos pedagógicos al uso, centrados con frecuencia de forma preferible en dar información y/o instrucción a los profesionales de la educación.

Una consecuencia de esa naturaleza de los textos propuestos para la lectura de los educadores es la discreta y delicada misión de quien ayuda a los lectores. A diferencia de una pedagogía marcadamente académica, en donde se les anticipa a quienes han de aprender expectativas, objetivos y formatos bien predeterminados, en el tipo de lectura que andamos tratando es suficiente con estimular en los lectores una actitud de escucha, de inquietud y de apertura; es decir, sugerir delicadamente algunas insinuaciones "que hagan que la lectura como experiencia sea posible" (Larrosa, 33). Eso es, precisamente, lo que intentamos hacer unos meses atrás con los profesores que decidieron leer el consabido libro utilizado para realizar la modesta investigación que hemos realizado y comentado.

Por lo que se refiere a las conclusiones particulares de este estudio, una de las más destacables es que, concretamente en la experiencia lectora de los textos fenomenológicos sugeridos, éstos han actuado a modo de espejos en los que los lectores (educadores) han podido leerse a si mismos; o como viene a decir Van Manen, generalizando: partiendo de las experiencias vicarias narradas en dichos textos, los profesores han tenido la posibilidad de ver o descubrir lo que es correcto o posible en sus propias experiencias educativas llevadas a cabo en la práctica diaria. En suma: los educadores tienen la posibilidad de ejercitar en ese tipo de lecturas una "reflexión solícita" sobre sus experiencias pasadas, adquiriendo así la sensibilidad propia del tacto y de la solicitud pedagógica $(1998,213)$.

Con el fin de poner, por ahora, punto y final a todo lo que se podría decir más matizadamente en función de lo que los profesores lectores han comentado en sus escritos, únicamente insistiré en que la aguda cuestión apuntada por Heidegger: "Puede la fenomenología hacer algo "con" nosotros?" se puede contestar de forma claramente afirmativa. Puede decirse, con certeza, que todos los educadores de nuestra muestra, cada uno de forma diferente, han suscrito que la lectura del contenido de los textos fenomenológicos ofrecidos en esta ocasión les

16. VAN MANEN hace referencia aquí a lo que GADAMER comenta en Verdad y método (1975), centrándose especialmente en las "experiencias de verdad estéticas" (ver, entre otras, las páginas primeras de su libro, 23-27).

17. Como dice en otro lugar VAN MANEN: "Al trasladar a la conciencia reflexiva la naturaleza de los acontecimientos experimentados en nuestra actitud natural, podemos trans-formarnos o re-hacernos, en el sentido auténtico de Bildung (formación)" (2003, 25). 
ha hecho cambiar muchos esquemas y actitudes pedagógicas, les ha esclarecido y despertado su conciencia educadora, les ha hecho sentirse más comprometidos profesional y éticamente en su relación con los alumnos, y les ha suscitado la aspiración de ser verdaderos educadores: desde los sencillos detalles de la práctica, pero orientados hacia el horizonte de la excelencia pedagógica. Estos profesores, en fin, han "mostrado" que, realmente, la lectura de este tipo de textos --iojalá hubiera gran abundancia de ellos!- tiene una notoria potencialidad formativa en el campo pedagógico, especialmente en el plano más actitudinal, humano y profundo.

\section{BiBLIOGRAFÍA}

Ayala, R. (2007) Introducción a la concepción y aplicación del método fenomenológico-hermenéutico de M. Van Manen. Universidad Autónoma de Barcelona. Trabajo de Investigación dirigido por el profesor José Antonio Jordán.

Bachelard, G. (1965) La poética del espacio. México, FCE.

BARnaCle, R. (2004) Reflection on Lived Experience in Educational Research, Educational Philosophy and Theory, 36 (1), 57-67.

BuYTENDijK, J. F. (1961) La psicologia de la novela: estudios sobre Dostoyeski. Buenos Aires, Editorial Lohlé.

HANSEn, D. T. (2001) Llamados a enseñar. Barcelona, Idea-Books.

LARrosa, J. (1996) La experiencia de la lectura. Estudios sobre literatura y formación. Barcelona, Laertes.

- (2003a) La experiencia de la lectura: estudios sobre literatura y formación. México, FCE.

- (2003b) Entre las lenguas: lenguaje y educación después de Babel. Barcelona, Laertes.

Gadamer, H. G. (1975) Verdad y método, vol. 1. Salamanca, Sígueme.

Levering, B. y Van Manen, M. (1999) Los secretos de la infancia. Barcelona, Paidós.

Merleau-Ponty, M. (1975) Fenomenología de la percepción. Barcelona, Península.

Proust, M. (1986) El temps retrobat. Barcelona, Edicions 62.

SAINT-EXupÉRY, A. de (1997) El principito. Madrid, Alianza Editorial.

Spiegelberg, H. (1982) The Phenomenological Movement. A Historical Introduction. La Haya: Nijhoff.

VANDENBerg, D. (1992) Researching, Lived Experience: A Review Essay, Educational Theory, $42(1), 119-126$.

VAn Manen, M. (1978) An experiment in educational theorizing: The Utrecht school, Interchange, 10 (1), 48-66.

- (1985) Phenomenology of the Novel, or How Do Novels teach?, Phenomenology and Pedagogy, 3 (23), 177-187.

- (1996) Phenomenological Pedagogy and the Question of Meaning, en Vandenberg, D. (ed.). Phenomenology and Educational Discourse. Durban, Heineman Higher and Further Education, 39-64.

- (1997) From Meaning to Method, Qualitative Health Research, 7 (3), 345-369.

- (1998) El tacto en la enseñanza. El significado de la sensibilidad pedagógica. Barcelona, Paidós. 
JOSÉ ANTONIO JORDÁN SIERRA

POTENCIALIDAD FORMATIVA DE LA LECTURA DE TEXTOS FENOMENOLÓGICOS

DESDE LA PERSPECTIVA DE VAN MANEN

- (2003) Investigación educativa y experiencia vivida. Barcelona, Idea-Books.

- (2004) El tono en la enseñanza. Barcelona, Paidós.

- (2007) Phenomenology of Practice, Phenomenology \& Practice, 1 (1), 11-30.

Van Manen, M. y Levering, B. (2002) Phenomenological Anthropology in the Netherlands and Flanders, en Tymieniecka, T. (ed.). Phenomenology World-Wide. Dordrecht, Kluwer Press, 274-286.

Van Manen, M. y Li, S. (2002) The pathic principle of pedagogical language, Teaching and Teacher Education, 18 (2), 215-224.

Web (páginas) consultadas

http://www.phenomenologyonline.com/Max/(web de Van Manen).

http://www.phenomenologyonline.com/inquiry/2.html.

http://www.phenomenologyonline.com/inquiry/8.html.

http://www.phenomenologyonline.com/inquiry/68.html.

http://phandpr.org/index.php/pandp/index (web de la revista Phenomenoloy \& Practice). 